\title{
Application of Root and Rhizome of Chinese Traditional Medicine Flowers
}

\author{
Ming Bai, Bingjie Chang, and Mingsan Miao* \\ Henan University of Traditional Chinese Medicine \\ Zhengzhou, China \\ miaomingsan@163.com
}

\begin{abstract}
To explore the application of flowers of medicinal herbs with root and rhizome plant as medicinal materials. Method: Through the literature review, Pharmacopoeia and related research background, analysis of root and rhizome flower's medicinal applications is investigated. Result: Root medicine is used widely in Chinese herbal medicine. Out of 521 kinds of traditional Chinese medicines in the manual of Chinese medicinal herbs, 121 kinds of root medicines are there [1]. In these plants, both root and rhizome are used for their propagation and flowers which are rich in nutrients, are mostly discarded, resulting in a huge waste of resources. Conclusion: This paper expounds the comprehensive utilization of root and rhizome of plant flowers, in order to provide theoretical basis for the best use of resources and protection of the natural environment.
\end{abstract}

Keywords-Rhizome, plant, flower, comprehensive utilization

Many species of root and rhizome are dominant and strong varieties [2]. In the process of production, the flowers are often discarded as waste. In fact, flowers not only have ornamental value, but also have the value of health care. They belong to traditional Chinese medicine. They play an important role in the fields of diet, medicine, beauty and healthcare [3]. In this paper, the main chemical constituents and pharmacological effects of root and rhizome plant flowers are presented, and their development and utilization value also will be discussed, which provides reference for further research and development.

\section{THE FLOWERS OF RESEARCH STATUS OF ROOT (RHIZOME) TRADITIONAL CHINESE MEDICINE}

In medicinal plants, the flower absorbs the energy of the sun, is the essence of plants. Flowers being main characteristic of plants aid in plant identification [4] Modern research has focused on chemical components and pharmacology, chemical composition of flowers mostly containing flavonoids, volatile oil, terpenoids, with antioxidant, antibacterial and anti-inflammatory, antitumor, immune enhancement and analgesia action. Root and rhizome plants flower produce of a variety of forms, and such flowers find use in the food, in the wine, tea, cosmetics, sweet bursa, flowers of dew, oil, etc, and all they are an important part of traditional culture in our country.

\section{A COMPREHENSIVE UTILIZATION OF ROOT (RHIZOME) TRADITIONAL CHINESE MEDICINE}

Roots and rhizomes of Chinese medicinal herbs account for a large proportion of Chinese medicinal herbs, and their flowers have some applications in modern studies. The application of flowers of root and rhizome medicinal was described in the following table. According to the characteristics of the tropism of sex and taste, the classification is carried out, and most of them are warm and flat. See table 1.

TABLE 1. APPLICATION OF ROOT AND RHIZOME PLANT FLOWERS

\begin{tabular}{|c|c|c|c|c|c|c|}
\hline Plant & $\begin{array}{l}\text { Tropi } \\
\text { sm of } \\
\text { Taste }\end{array}$ & $\begin{array}{l}\text { Chemical } \\
\text { Composition }\end{array}$ & $\begin{array}{l}\text { Pharmacolo } \\
\text { Gical action }\end{array}$ & Funtion Indicated & Dosege & Food Therapy \\
\hline $\begin{array}{l}\text { Achnat } \\
\text { herum } \\
\text { splend } \\
\text { ens } \\
\text { flower } \\
\text { s }\end{array}$ & $\begin{array}{l}\text { gan } \\
\text { ping }\end{array}$ & Flavonoids & & $\begin{array}{l}\text { Diuresis,hemostasis.Indications } \\
\text { mewborn urine adverse,internal bleeding }\end{array}$ & $\begin{array}{l}\text { Decoct } \\
\text { soup } 15 \sim 30 \\
\mathrm{~g}\end{array}$ & \\
\hline $\begin{array}{l}\text { Impera } \\
\text { ta } \\
\text { cylindr } \\
\text { ica } \\
\text { flower } \\
\mathrm{s}\end{array}$ & $\begin{array}{l}\text { gan } \\
\text { light } \\
\text { ping }\end{array}$ & Triterpene & $\begin{array}{l}\text { Blood } \\
\text { coagulation } \\
\text { and } \\
\text { hemostasis }\end{array}$ & $\begin{array}{l}\text { Hemostasis,detoxification,pain.Indications } \\
\text { hematemesis,nose bleed,stab wounds }\end{array}$ & $\begin{array}{l}\text { Decoct } \\
\text { soup9 15g }\end{array}$ & $\begin{array}{l}\text { Epistaxis:flowers five money,a } \\
\text { pig nose }\end{array}$ \\
\hline $\begin{array}{l}\text { Cycads } \\
\text { flower } \\
\text { s }\end{array}$ & $\begin{array}{l}\text { gan } \\
\text { ping }\end{array}$ & Flavonoids & & $\begin{array}{l}\text { Qi dampness,stop blood, Yishen } \\
\text { Guijing.IndicationsofStomachache,chronicHepat } \\
\text { itis,hematemesis,dysmenorrhea,sprmatorrhea } \\
\text { vaginal discharge }\end{array}$ & $\begin{array}{l}\text { Decoct } \\
\text { soup } 15 \sim 60 \\
\mathrm{~g}\end{array}$ & A little posion \\
\hline $\begin{array}{l}\text { Pine } \\
\text { flower } \\
\text { s }\end{array}$ & $\begin{array}{l}\text { sweet } \\
\text { warm } \\
\text { liver } \\
\text { stom } \\
\text { ach }\end{array}$ & Selenium & $\begin{array}{l}\text { Antitum } \\
\text { orThe role of } \\
\text { the immune } \\
\text { system }\end{array}$ & $\begin{array}{l}\text { Expelling wind,supplementing qi,colecting } \\
\text { dampness and enriching blood Indications } \\
\text { headacha dizziness,diarrhea,eczema wet } \\
\text { sores,tranma bleeding }\end{array}$ & $\begin{array}{l}\text { Fried } \\
\text { soup3 9g; } \\
\text { or a blunt }\end{array}$ & \\
\hline
\end{tabular}


TABLE 1. APPLICATION OF ROOT AND RHIZOME PLANT FLOWERS

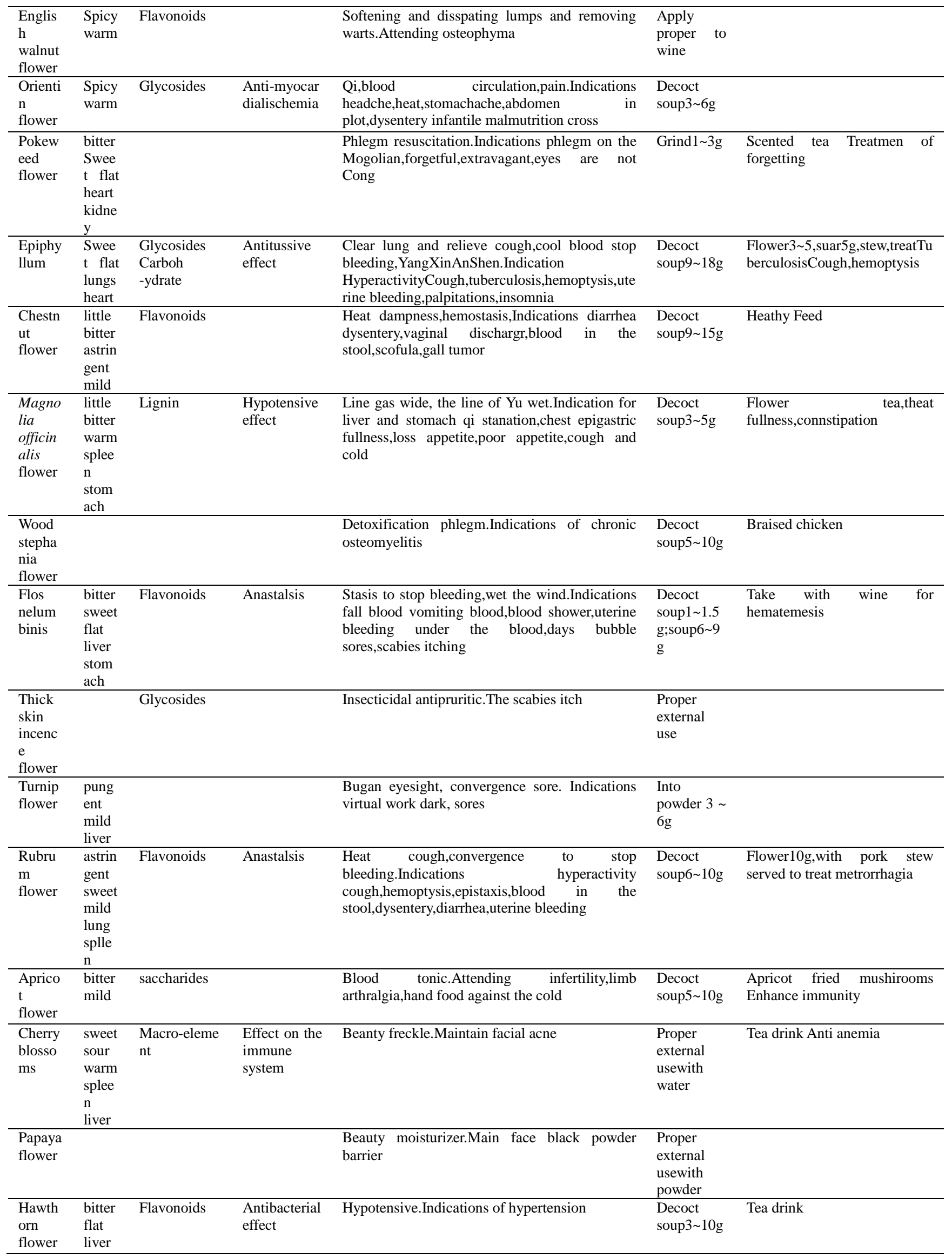


TABLE 1. APPLICATION OF ROOT AND RHIZOME PLANT FLOWERS

\begin{tabular}{|c|c|c|c|c|c|c|}
\hline $\begin{array}{l}\text { Loquat } \\
\text { flower }\end{array}$ & $\begin{array}{l}\text { Light } \\
\text { flat } \\
\text { merid } \\
\text { ian }\end{array}$ & $\begin{array}{l}\text { Flavonoids } \\
\text { amygdalin }\end{array}$ & $\begin{array}{l}\text { Relieve } \\
\text { cough } \\
\text { Antisepsis } \\
\text { and } \\
\text { anti-inflamm } \\
\text { atory effects }\end{array}$ & $\begin{array}{l}\text { Expelling wind and relieving cough and } \\
\text { obstruction of nose.The theatment of common } \\
\text { cold cough,nasal mucus,asthenia long } \\
\text { cough,bloody sputum }\end{array}$ & $\begin{array}{l}\text { Decoct } \\
\text { soup6 12g }\end{array}$ & $\begin{array}{l}\text { Stewed with } \\
\text { chicken.Nourishing Yinmoiste } \\
\text { n,phlegm cough }\end{array}$ \\
\hline $\begin{array}{l}\text { Soft } \\
\text { Palm } \\
\text { flower }\end{array}$ & $\begin{array}{l}\text { bitter } \\
\text { astrig } \\
\text { ent } \\
\text { mild }\end{array}$ & & & Hemostasis.Indications for bleeding & $\begin{array}{l}\text { Decoct } \\
\text { soup9 15g }\end{array}$ & \\
\hline $\begin{array}{l}\text { Arunc } \\
\text { us } \\
\text { flower }\end{array}$ & $\begin{array}{l}\text { Astri } \\
\text { gent } \\
\text { mild } \\
\text { liver } \\
\text { stom } \\
\text { ach } \\
\text { splee } \\
\text { n }\end{array}$ & Glycosides & $\begin{array}{l}\text { Diuretic } \\
\text { action }\end{array}$ & Antituberculosis. Treating tuiberculosis & $\begin{array}{l}\text { Decoct } \\
\text { soup6 12g }\end{array}$ & $\begin{array}{l}\text { Kerria japontca steamed honey } \\
\text { Ralieve a cough }\end{array}$ \\
\hline $\begin{array}{l}\text { Rose } \\
\text { flower }\end{array}$ & $\begin{array}{l}\text { sweet } \\
\text { flat }\end{array}$ & & & $\begin{array}{l}\text { Moistening lung to stop cough. } \\
\text { Indications feilao cough }\end{array}$ & $\begin{array}{l}\text { Decoct } \\
\text { soup6 15g }\end{array}$ & \\
\hline $\begin{array}{l}\text { Dahuri } \\
\text { an rose } \\
\text { flower }\end{array}$ & $\begin{array}{l}\text { slight } \\
\text { ly } \\
\text { sour } \\
\text { bitter, } \\
\text { mild }\end{array}$ & Volayile oil & Antiviral & $\begin{array}{l}\text { Regularting qi,relieving cough.Treating irregular } \\
\text { menstruation,dysmenorrhea, } \\
\text { uterine bleeding,hematemesis,inter costal } \\
\text { neuralgia,feilao cough }\end{array}$ & $\begin{array}{l}\text { Decoct } \\
\text { soup3 6g }\end{array}$ & $\begin{array}{l}\text { Tea drink. } \\
\text { Invigorate the circulation of } \\
\text { blood }\end{array}$ \\
\hline $\begin{array}{l}\text { Cherok } \\
\text { ee rose } \\
\text { flower }\end{array}$ & $\begin{array}{l}\text { sour } \\
\text { flat }\end{array}$ & Volayile oil & $\begin{array}{l}\text { Antibacterial } \\
\text { action. Anti } \\
\text { atheroscleros } \\
\text { is }\end{array}$ & $\begin{array}{l}\text { Astringent intestinal,solid fine,insecticide. } \\
\text { Indications JiuxieJiuli,nocturnal emossion, } \\
\text { urinary frequency,enuresis,vaginal } \\
\text { discharge,worm disease,send early white }\end{array}$ & $\begin{array}{l}\text { Decoct } \\
\text { soup3 9g }\end{array}$ & Tea drink \\
\hline $\begin{array}{l}\text { Sachali } \\
\mathrm{n} \\
\text { raspber } \\
\text { ry leaf } \\
\text { flower }\end{array}$ & $\begin{array}{l}\text { bitter } \\
\text { flat }\end{array}$ & & & $\begin{array}{l}\text { Detoxification } \\
\text { Indications snake scorpion bites, soothe the } \\
\text { nerves }\end{array}$ & $\begin{array}{l}\text { Decoct } \\
\text { soup3 10g } \\
; \\
\text { Proper } \\
\text { external } \\
\text { use }\end{array}$ & \\
\hline $\begin{array}{l}\text { Flower } \\
\text { of } \\
\text { silktree } \\
\text { albizzi } \\
\text { a }\end{array}$ & $\begin{array}{l}\text { sweet } \\
\text { bitter } \\
\text { flat } \\
\text { heart } \\
\text { splee } \\
\mathrm{n} \\
\end{array}$ & $\begin{array}{l}\text { Aromatic } \\
\text { components }\end{array}$ & $\begin{array}{l}\text { Central } \\
\text { inhibitory } \\
\text { effect }\end{array}$ & $\begin{array}{l}\text { To relieve god, air, appetizer, wind, and blood. } \\
\text { Treatment of insomnia, anorexia, depression, } \\
\text { chest heat eye problems, blurred vision, pain, } \\
\text { injury. }\end{array}$ & $\begin{array}{l}\text { Decoct } \\
\text { soup3 9g }\end{array}$ & $\begin{array}{l}\text { Flos albiziae porridge } \\
\text { Nerves resolve depression }\end{array}$ \\
\hline $\begin{array}{l}\text { Franch } \\
\text { et } \\
\text { peashr } \\
\text { ub } \\
\text { flower }\end{array}$ & $\begin{array}{l}\text { sweet } \\
\text { bitter } \\
\text { mild }\end{array}$ & $\begin{array}{l}\text { Alkaloid } \\
\text { Glycosides }\end{array}$ & $\begin{array}{l}\text { Hypotensive } \\
\text { effect }\end{array}$ & $\begin{array}{l}\text { Invigorate the kidney and } \\
\text { spleen.Dizziness, headache,tinnitus,Indications } \\
\text { kidneg feilao cough,infantile malnutrition }\end{array}$ & $\begin{array}{l}\text { Decoct } \\
\text { soup3 9g }\end{array}$ & $\begin{array}{l}\text { Steanmed egg roll.Invigorate } \\
\text { the spleen and kidney }\end{array}$ \\
\hline $\begin{array}{l}\text { Interm } \\
\text { ediate } \\
\text { peashr } \\
\text { ub } \\
\text { flower }\end{array}$ & $\begin{array}{l}\text { sweet } \\
\text { warm }\end{array}$ & & & $\begin{array}{l}\text { Nourishing Yin to calm the liver.Indications } \\
\text { dizziness, hypertension }\end{array}$ & $\begin{array}{l}\text { Decoct } \\
\text { soup6 15g }\end{array}$ & $\begin{array}{l}\text { Intermediate peashrub flower } \\
\text { tea }\end{array}$ \\
\hline $\begin{array}{l}\text { Caraga } \\
\text { na } \\
\text { Sinica } \\
\text { flower }\end{array}$ & $\begin{array}{l}\text { sweet } \\
\text { tepid }\end{array}$ & & & $\begin{array}{l}\text { Relieving cough, stasis and pain,dispelling } \\
\text { wind.Indications of lung cough,infantile } \\
\text { malnutrition,liver Yang headache and } \\
\text { dizziness,traumatic injuiy }\end{array}$ & $\begin{array}{l}\text { Decoct } \\
\text { soup3 9g }\end{array}$ & \\
\hline $\begin{array}{l}\text { Flower } \\
\text { of } \\
\text { Chines } \\
\text { e } \\
\text { redbud }\end{array}$ & $\begin{array}{l}\text { bitter } \\
\text { mold }\end{array}$ & $\begin{array}{l}\text { Flavonoids } \\
\text { Glycosides }\end{array}$ & & $\begin{array}{l}\text { Clearing away heat and cooling blood and } \\
\text { detoxicating.Indications drench,sores blood in } \\
\text { treating rheumatism pain }\end{array}$ & $\begin{array}{l}\text { Decoct } \\
\text { soup3 6g }\end{array}$ & Tea drink \\
\hline $\begin{array}{l}\text { Lablab } \\
\text { flos }\end{array}$ & $\begin{array}{l}\text { sweet } \\
\text { mild }\end{array}$ & $\begin{array}{l}\text { Anthocyanin } \\
\text { us } \\
\text { Flavonoids }\end{array}$ & $\begin{array}{l}\text { Antibacterial } \\
\text { action }\end{array}$ & $\begin{array}{l}\begin{array}{l}\text { Summerheat dampness in the spleen. The } \\
\text { attending summer }\end{array} \\
\text { heat } \\
\text { dampness,fever,diarrhea,dysentery,vaginal } \\
\text { discharge,bone bruise }\end{array}$ & $\begin{array}{l}\text { Decoct } \\
\text { soup3 9g }\end{array}$ & Tea drink \\
\hline $\begin{array}{l}\text { Lesped } \\
\text { eza } \\
\text { flower }\end{array}$ & $\begin{array}{l}\text { sweet } \\
\text { mild }\end{array}$ & & & $\begin{array}{l}\text { Clear heat and stop bleeding,moistening lung } \\
\text { and relieving cough,the blood in the stool,lung } \\
\text { heat cough }\end{array}$ & $\begin{array}{l}\text { Decoct } \\
\text { soup9 15g }\end{array}$ & \\
\hline
\end{tabular}


TABLE 1. APPLICATION OF ROOT AND RHIZOME PLANT FLOWERS

\begin{tabular}{|c|c|c|c|c|c|c|}
\hline $\begin{array}{l}\text { Escobi } \\
\text { ta }\end{array}$ & $\begin{array}{l}\text { sweet } \\
\text { mild }\end{array}$ & & & $\begin{array}{l}\text { Clear heat and cool blood.Indications } \\
\text { cough,hematuria and hematochezia }\end{array}$ & $\begin{array}{l}\text { Decoct } \\
\text { soup30 60 } \\
\mathrm{g}\end{array}$ & \\
\hline $\begin{array}{l}\text { Crowt } \\
\text { oe } \\
\text { flower }\end{array}$ & $\begin{array}{l}\text { bitter } \\
\text { spicy } \\
\text { mild }\end{array}$ & Flavonoids & & $\begin{array}{l}\text { Clear heat and improve eyesight.Indications } \\
\text { anemopyretic red eyes,blurred vision }\end{array}$ & $\begin{array}{l}\text { Decoct } \\
\text { soup6 10g }\end{array}$ & \\
\hline $\begin{array}{l}\text { Kweic } \\
\text { how } \\
\text { milletti } \\
\text { a } \\
\text { flower }\end{array}$ & $\begin{array}{l}\text { sweet } \\
\text { astrin } \\
\text { gent } \\
\text { mild }\end{array}$ & & & $\begin{array}{l}\text { Hemostasis by convergence.Indications } \\
\text { epistaxis }\end{array}$ & $\begin{array}{l}\text { Decoct } \\
\text { soup6 9g }\end{array}$ & \\
\hline $\begin{array}{l}\text { Comm } \\
\text { on } \\
\text { averrh } \\
\text { oa } \\
\text { flower }\end{array}$ & $\begin{array}{l}\text { sweet } \\
\text { mind } \\
\text { smoo } \\
\text { th }\end{array}$ & $\begin{array}{l}\text { Quercetin } \\
\text { Glycosides }\end{array}$ & & $\begin{array}{l}\text { Cut off,pain,detoxification,insecticide } \\
\text { Indications malaria,stomach pain,paint a scabies }\end{array}$ & $\begin{array}{l}\text { Decoct } \\
\text { soup9 30g }\end{array}$ & \\
\hline $\begin{array}{l}\text { Pumm } \\
\text { elo } \\
\text { flower }\end{array}$ & $\begin{array}{l}\text { spicy } \\
\text { bitter } \\
\text { warm } \\
\text { lung } \\
\text { stom } \\
\text { ach } \\
\end{array}$ & $\begin{array}{l}\text { Volayile oil } \\
\text { Flavonoids }\end{array}$ & $\begin{array}{l}\text { Anti-inflam } \\
\text { matory and } \\
\text { antibacterial } \\
\text { Antitumor } \\
\text { Antioxidatio } \\
\mathrm{n}\end{array}$ & $\begin{array}{l}\text { Promoting Qi,resolveing phlefm and relieving } \\
\text { pain.Chest pain,epigastric indications }\end{array}$ & $\begin{array}{l}\text { Decoct } \\
\text { soup1.5 4. } \\
5 \mathrm{~g}\end{array}$ & $\begin{array}{l}\text { Flower boiled pork stomach } \\
\text { qi,spleen and stomach }\end{array}$ \\
\hline $\begin{array}{l}\text { Panicu } \\
\text { lata } \\
\text { flower }\end{array}$ & $\begin{array}{l}\text { spicy } \\
\text { bitter } \\
\text { warm }\end{array}$ & $\begin{array}{l}\text { Volayile oil } \\
\text { Carbohydrate } \\
\text { s }\end{array}$ & $\begin{array}{l}\text { Analgesic } \\
\text { effect }\end{array}$ & $\begin{array}{l}\text { Regulating Qi to relieve pain.Indications qi } \\
\text { stagnation anf stomache pain }\end{array}$ & $\begin{array}{l}\text { Decoct } \\
\text { soup3 9g }\end{array}$ & $\begin{array}{l}\text { Porridge. Treat tramayic } \\
\text { swelling,pain }\end{array}$ \\
\hline $\begin{array}{l}\text { Flos } \\
\text { longan }\end{array}$ & $\begin{array}{l}\text { slight } \\
\text { ly } \\
\text { bitter } \\
\text { sweet }\end{array}$ & $\begin{array}{l}\text { Phenolic } \\
\text { Carboxylic } \\
\text { acid } \\
\text { Flavonoids }\end{array}$ & $\begin{array}{l}\text { Hypoglycem } \\
\text { ic } \\
\text { effect }\end{array}$ & $\begin{array}{l}\text { Clear heat and toxic herbs ans arrest } \\
\text { sores.Indications }\end{array}$ & $\begin{array}{l}\text { Decoct } \\
\text { soup9 15g }\end{array}$ & Stew with pork \\
\hline $\begin{array}{l}\text { Comm } \\
\text { on } \\
\text { euscap } \\
\text { his } \\
\text { flower }\end{array}$ & $\begin{array}{l}\text { sweet } \\
\text { flat }\end{array}$ & & & $\begin{array}{l}\text { Dispelling wind and relieving } \\
\text { pain.headache.dizziness }\end{array}$ & $\begin{array}{l}\text { Decoct } \\
\text { soup10 15 } \\
\mathrm{g}\end{array}$ & \\
\hline $\begin{array}{l}\text { Phoeni } \\
\text { x tree } \\
\text { flower }\end{array}$ & $\begin{array}{l}\text { sweet } \\
\text { flat }\end{array}$ & $\begin{array}{l}\text { Volayile oil } \\
\text { Polycscchari } \\
\text { d }\end{array}$ & $\begin{array}{l}\text { Hypotensive } \\
\text { effect }\end{array}$ & $\begin{array}{l}\text { Dampness,detoxification.the } \\
\text { edema,dysuria,innominate } \\
\text { swelling,redness,tinea capitis,burn wound }\end{array}$ & $\begin{array}{l}\text { Decoct } \\
\text { soup6 15g }\end{array}$ & $\begin{array}{l}\text { Juice } \\
\text { Mumps treatment }\end{array}$ \\
\hline $\begin{array}{l}\text { Pomeg } \\
\text { ranate } \\
\text { flower }\end{array}$ & $\begin{array}{l}\text { sour } \\
\text { astrin } \\
\text { gent } \\
\text { mild }\end{array}$ & Volayile oil & $\begin{array}{l}\text { Anti-oxidati } \\
\text { on } \\
\text { Hypoglycem } \\
\text { ic }\end{array}$ & $\begin{array}{l}\text { Cool blood and stop bleeding.Indications } \\
\text { Nvxue,hematemesis,traumatic bleeding, } \\
\text { irregular menstruation,leucorrhea,red collapse } \\
\text { otitis media }\end{array}$ & $\begin{array}{l}\text { Decoct } \\
\text { soup3 6g }\end{array}$ & Tea drink for toothache \\
\hline $\begin{array}{l}\text { Chines } \\
\mathrm{e} \\
\text { alangiu } \\
\mathrm{m} \\
\text { flower }\end{array}$ & $\begin{array}{l}\text { spicy } \\
\text { natur } \\
\text { ed } \\
\text { liver } \\
\text { stom } \\
\text { ach } \\
\end{array}$ & Alkaloids & $\begin{array}{l}\text { Antibacterial } \\
\text { effect }\end{array}$ & $\begin{array}{l}\text { Dispersing wind, regulating qi andrelieving } \\
\text { pain.Indications headache and abdominal pain }\end{array}$ & $\begin{array}{l}\text { Decoct } \\
\text { soup3 10g }\end{array}$ & Small poison \\
\hline $\begin{array}{l}\text { Aralia } \\
\text { flower }\end{array}$ & $\begin{array}{l}\text { bitter } \\
\text { satrin } \\
\text { gent } \\
\text { mild }\end{array}$ & Steroids & $\begin{array}{l}\text { Anti-inflam } \\
\text { matory } \\
\text { effect }\end{array}$ & Hemostasis.Indications for hematemesis & $\begin{array}{l}\text { Decoct } \\
\text { soup9 15g }\end{array}$ & \\
\hline $\begin{array}{l}\text { Ginsen } \\
\mathrm{g} \\
\text { flower }\end{array}$ & $\begin{array}{l}\text { spicy } \\
\text { tepid } \\
\text { heart } \\
\text { kidne } \\
\text { y }\end{array}$ & $\begin{array}{l}\text { Ginsenosides } \\
\text { Polysacchari } \\
\text { des }\end{array}$ & $\begin{array}{l}\text { Anti-ulcer } \\
\text { effect } \\
\text { Anti-tumor } \\
\text { effect } \\
\text { Anti-aging }\end{array}$ & $\begin{array}{l}\text { Invigorating qi and strengthening the body and } \\
\text { delaying senility }\end{array}$ & $\begin{array}{l}\text { Make } \\
\text { tea3 6g }\end{array}$ & $\begin{array}{l}\text { walnut,lean meat soup.Ziyi } \\
\text { Bushen,strong } \\
\text { vitality for men Adjust blood, } \\
\text { moisturizing hair for women }\end{array}$ \\
\hline $\begin{array}{l}\text { Rice } \\
\text { paper } \\
\text { plant } \\
\text { immat } \\
\text { ure } \\
\text { flower }\end{array}$ & $\begin{array}{l}\text { sweet } \\
\text { mild }\end{array}$ & & & $\begin{array}{l}\text { Smoothing the liver to promote qi } \\
\text { Indications hemia }\end{array}$ & $\begin{array}{l}\text { Decoct } \\
\text { soup30 60 } \\
\mathrm{g}\end{array}$ & \\
\hline $\begin{array}{l}\text { Divari } \\
\text { cate } \\
\text { saposh } \\
\text { nikovia } \\
\text { flower }\end{array}$ & $\begin{array}{l}\text { spicy } \\
\text { tepid } \\
\text { liver } \\
\text { splee } \\
\mathrm{n} \\
\text { stom } \\
\text { ach }\end{array}$ & & & $\begin{array}{lcc}\text { Regulating } & \text { qi to relieve } & \text { pain.Indication } \\
\text { abdominal } & \text { werwantong,joint } & \text { pain,spasm of } \\
\text { limbs } & & \end{array}$ & $\begin{array}{l}\text { Decoct } \\
\text { soup3 6g }\end{array}$ & \\
\hline
\end{tabular}


TABLE 1. APPLICATION OF ROOT AND RHIZOME PLANT FLOWERS

\begin{tabular}{|c|c|c|c|c|c|c|}
\hline $\begin{array}{l}\text { Persim } \\
\text { mon } \\
\text { flower }\end{array}$ & $\begin{array}{l}\text { sweet } \\
\text { mild } \\
\text { lung } \\
\text { splee } \\
\mathrm{n}\end{array}$ & & & $\begin{array}{l}\text { Reducing qi and stomoch,detoxification and } \\
\text { convergence.Indications a Tunsuan,vomiting }\end{array}$ & $\begin{array}{l}\text { Decoct } \\
\text { soup3 6g }\end{array}$ & $\begin{array}{l}\text { Treat hiccup by decocting } \\
\text { flower }\end{array}$ \\
\hline $\begin{array}{l}\text { Caudat } \\
\text { e sweet } \\
\text { leaf } \\
\text { flower }\end{array}$ & $\begin{array}{l}\text { bitter } \\
\text { spicy } \\
\text { flat } \\
\text { merid } \\
\text { ian } \\
\end{array}$ & Saponins & & $\begin{array}{l}\text { A phlegm and a quenching thirst. Indications } \\
\text { cough chest tightness, children diarrhea }\end{array}$ & $\begin{array}{l}\text { Decoct } \\
\text { soup6 9g }\end{array}$ & Treat cough and chest distress \\
\hline $\begin{array}{l}\text { Clerod } \\
\text { endron } \\
\text { yunnan } \\
\text { ensis } \\
\text { hu } \\
\text { flower }\end{array}$ & $\begin{array}{l}\text { slight } \\
\text { ly } \\
\text { bitter } \\
\text { warm }\end{array}$ & Alkaloids & & $\begin{array}{l}\text { Hemostasis. Indications uterine bleeding, } \\
\text { vaginal diacharge }\end{array}$ & $\begin{array}{l}\text { Decoct } \\
\text { soup } 10 \sim 15 \\
\mathrm{~g}\end{array}$ & \\
\hline $\begin{array}{l}\text { Wild } \\
\text { sesame } \\
\text { seed } \\
\text { flower }\end{array}$ & $\begin{array}{l}\text { sweet } \\
\text { spicy } \\
\text { mild }\end{array}$ & & & $\begin{array}{l}\text { Blood circulation, cooling blood heat. } \\
\text { Indications irregular menstruation, } \\
\text { dysmenorrhea, chiba taginal discharg, } \\
\text { ehyperactivity hemoptysis, urine pain }\end{array}$ & $\begin{array}{l}\text { Decoct } \\
\text { soup10 25 } \\
\mathrm{g}\end{array}$ & \\
\hline $\begin{array}{l}\text { Garden } \\
\text { eggpla } \\
\text { nt } \\
\text { flower }\end{array}$ & $\begin{array}{l}\text { sweet } \\
\text { flat }\end{array}$ & & & $\begin{array}{l}\text { Convergence sores,pain dampness. } \\
\text { Indications truma,toothache,women with } \\
\text { excessive vaginal discharge }\end{array}$ & $\begin{array}{l}\text { Powder2 3 } \\
\mathrm{g}\end{array}$ & $\begin{array}{l}\text { Tea drink } \\
\text { Treat toochache }\end{array}$ \\
\hline $\begin{array}{l}\text { Elder } \\
\text { flower }\end{array}$ & $\begin{array}{l}\text { spisy } \\
\text { warm }\end{array}$ & Glycosides & $\begin{array}{l}\text { Anti-inflam } \\
\text { matory } \\
\text { effect }\end{array}$ & Sweeting diuresus. Indications cold, urine aderse & $\begin{array}{l}\text { Decoct } \\
\text { soup4.5 9 } \\
\mathrm{g}\end{array}$ & $\begin{array}{l}\text { Tea drink } \\
\text { Treat perdpiration }\end{array}$ \\
\hline $\begin{array}{l}\text { Potmar } \\
\text { igold } \\
\text { calend } \\
\text { ula } \\
\text { flower }\end{array}$ & $\begin{array}{l}\text { flat } \\
\text { level }\end{array}$ & $\begin{array}{l}\text { Carbohydrate } \\
\text { s steroids }\end{array}$ & $\begin{array}{l}\text { Hypolopide } \\
\text { mic effect } \\
\text { Effect on the } \\
\text { immune } \\
\text { system }\end{array}$ & $\begin{array}{l}\text { Cooling blood to stop bleeding, heat purging } \\
\text { fire. Indications intestinal wind blood in the } \\
\text { stool, red eyes and throat }\end{array}$ & $\begin{array}{l}\text { Decoct } \\
\text { soup5 10g }\end{array}$ & $\begin{array}{l}\text { Theat intestinal wind and } \\
\text { blood in the stool }\end{array}$ \\
\hline $\begin{array}{l}\text { Cairo } \\
\text { mornin } \\
\text { g glory } \\
\text { flower }\end{array}$ & $\begin{array}{l}\text { slight } \\
\text { ly } \\
\text { bitter } \\
\text { warm }\end{array}$ & & & $\begin{array}{l}\text { Relieving cough and steaming. The deficiency of } \\
\text { consumption heat,cough hemorrhage }\end{array}$ & $\begin{array}{l}\text { Proper } \\
\text { amount use }\end{array}$ & Soup \\
\hline
\end{tabular}

There is a small part of cold, see table 2.

TABLE 2 THE FLOWER OF APPLOCATION OF ROOT AND RHIZOMA TRADITIONAL CHINESE MEDICINE

\begin{tabular}{|c|c|c|c|c|c|c|}
\hline Plant & $\begin{array}{l}\text { Tropism } \\
\text { of Taste }\end{array}$ & $\begin{array}{l}\text { Chemical } \\
\text { Composition }\end{array}$ & $\begin{array}{l}\text { Pharmacologi } \\
\text { cal Action }\end{array}$ & Function Indicated & Dosege & $\begin{array}{l}\text { Food } \\
\text { Therapy }\end{array}$ \\
\hline $\begin{array}{l}\text { Poplar } \\
\text { flower }\end{array}$ & $\begin{array}{l}\text { bitter } \\
\text { cold } \\
\text { large } \\
\text { intestine }\end{array}$ & Flavonoids & $\begin{array}{l}\text { The effect on } \\
\text { the immune } \\
\text { system }\end{array}$ & $\begin{array}{l}\text { Clear the heat and matetial, elimminating } \\
\text { dampness to stop dysentery. Indications } \\
\text { bacterial dysentery, enteritis }\end{array}$ & $\begin{array}{l}\text { Decoct } \\
\text { soup9 15g }\end{array}$ & \\
\hline $\begin{array}{l}\text { Ramie } \\
\text { flower }\end{array}$ & $\begin{array}{l}\text { sweet } \\
\text { cold }\end{array}$ & Hydrocysnic acid & Hemostasis & $\begin{array}{l}\text { Clear the heat and rash, relieve the cold. } \\
\text { Indications upset insomnia, mouth sores, } \\
\text { measles through the poor, rubella itching }\end{array}$ & $\begin{array}{l}\text { Decoct } \\
\text { soup6 15g }\end{array}$ & \\
\hline $\begin{array}{l}\text { Purple } \\
\text { jasmine } \\
\text { flower }\end{array}$ & $\begin{array}{l}\text { slighly } \\
\text { sweet } \\
\text { cool } \\
\text { lung }\end{array}$ & Glycosides & $\begin{array}{l}\text { Antibacterial } \\
\text { effect }\end{array}$ & $\begin{array}{l}\text { Run lung cooling blood. Indications } \\
\text { hemoptysis }\end{array}$ & $\begin{array}{l}\text { Decoct } \\
\text { soup60 12 } \\
0 \mathrm{~g}\end{array}$ & $\begin{array}{l}\text { Eat with tofu, } \\
\text { regularting } \\
\text { endocrine }\end{array}$ \\
\hline $\begin{array}{l}\text { Basellaceae } \\
\text { flower }\end{array}$ & $\begin{array}{l}\text { bitter } \\
\text { cold }\end{array}$ & Glycosides & & $\begin{array}{l}\text { Cool blood detoxification. Indications of } \\
\text { smallpox, nipple rupture }\end{array}$ & $\begin{array}{l}\text { Approptiat } \\
\text { e } \\
\text { amount }\end{array}$ & \\
\hline $\begin{array}{l}\text { Celosia } \\
\text { flower }\end{array}$ & $\begin{array}{l}\text { bitter } \\
\text { cold }\end{array}$ & & & & $\begin{array}{l}\text { Decoct } \\
\text { soup15 30 } \\
\mathrm{g}\end{array}$ & $\begin{array}{l}\text { Stew with } \\
\text { pork }\end{array}$ \\
\hline celosia & $\begin{array}{l}\text { sweet } \\
\text { astringe } \\
\text { nt cool }\end{array}$ & $\begin{array}{l}\text { Flavonoids } \\
\text { Anthocyanins }\end{array}$ & $\begin{array}{l}\text { Induction of } \\
\text { labor } \\
\text { Antiviral effect }\end{array}$ & $\begin{array}{l}\text { Cool blood to stop bleeding, only with } \\
\text { diarrhea. Indications bleeding, vaginal } \\
\text { diacharge, diarrhea, dysentery }\end{array}$ & $\begin{array}{l}\text { Decoct } \\
\text { soup9 15g }\end{array}$ & $\begin{array}{l}\text { With the } \\
\text { bird's nest } \\
\text { cockscomb } \\
\text { stew for } \\
\text { nourshing yin } \\
\text { formoistening } \\
\text { dryness }\end{array}$ \\
\hline
\end{tabular}


TABLE 2 THE FLOWER OF APPLOCATION OF ROOT AND RHIZOMA TRADITIONAL CHINESE MEDICINE

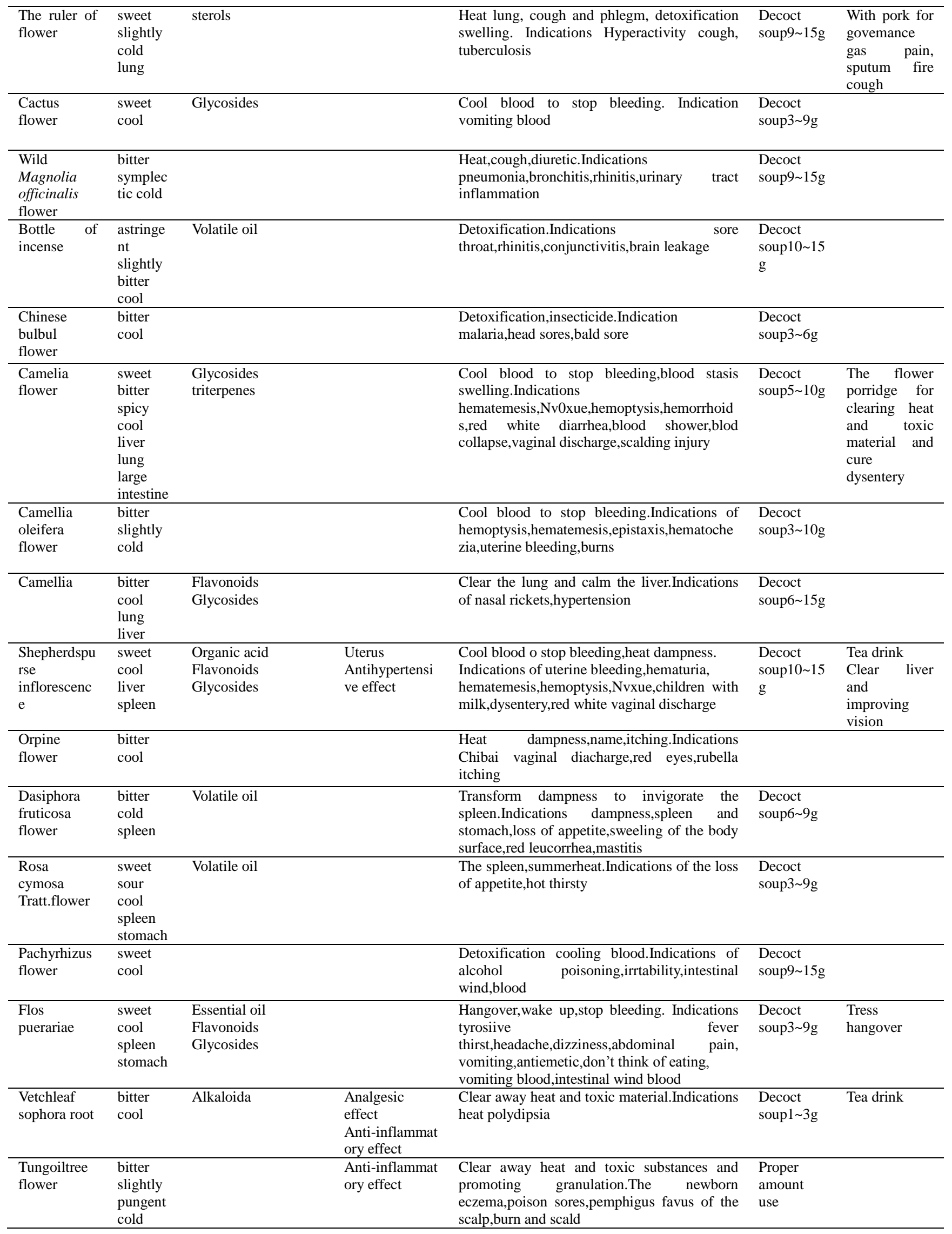


TABLE 2 THE FLOWER OF APPLOCATION OF ROOT AND RHIZOMA TRADITIONAL CHINESE MEDICINE

\begin{tabular}{|c|c|c|c|c|c|}
\hline $\begin{array}{l}\text { Chinese } \\
\text { sumac } \\
\text { flower }\end{array}$ & $\begin{array}{l}\text { salty } \\
\text { slightly } \\
\text { cold }\end{array}$ & & $\begin{array}{l}\text { Clear away heat and toxic herbs and arrest } \\
\text { sores.Indications sore for a long time } \\
\text { closing,the nose on both sides under the red } \\
\text { sores,itching,oozing into erosion }\end{array}$ & $\begin{array}{l}\text { Proper } \\
\text { amount } \\
\text { use }\end{array}$ & \\
\hline $\begin{array}{l}\text { Flower of } \\
\text { begonia }\end{array}$ & $\begin{array}{l}\text { bitter } \quad \text { Carbohydrate } \\
\text { acid }\end{array}$ & $\begin{array}{l}\text { Antibactetial } \\
\text { effect }\end{array}$ & $\begin{array}{l}\text { Insecticidal turbid channels. Indications for } \\
\text { tima pedis }\end{array}$ & $\begin{array}{l}\text { Proper } \\
\text { amount } \\
\text { use }\end{array}$ & $\begin{array}{l}\text { Begonia } \\
\text { flower } \\
\text { chestnut } \\
\text { congee } \\
\text { trumatic } \\
\text { injury }\end{array}$ \\
\hline
\end{tabular}

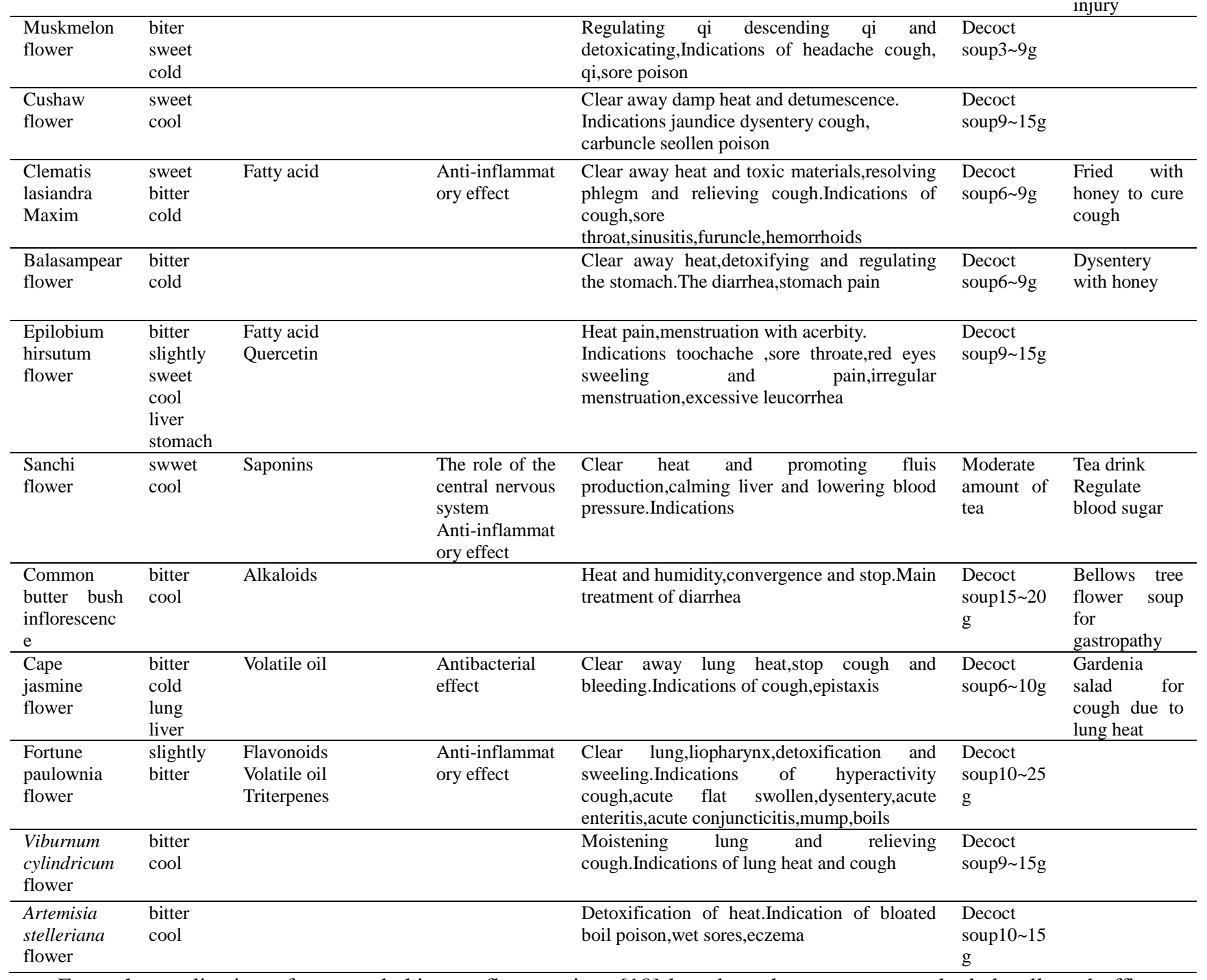

From the application of root and rhizome flowers, it seems that the 95 kinds of flowers which are not commonly used for are cold, hot, warm and cool (flat), and the four are warm and flat. In toxicity, there are only 2 kinds of big and small ones marked clearly. These flowers are not commonly used for resistance and [5-6], and find wide application for the light flexible products. Hence these flowers an important raw material for people, often with disease prevention and health care, to provide ideas for the [7-9] application root and rhizome medicinal flower.

\section{DISCUSSION}

In the 2015 edition of the "Chinese Pharmacopoeia"
[10] based on the source, spent herbal collected efficacy, taste, meridian, chemical composition, pharmacological effects were summarized, and to explore the therapeutic effect of flower into tea, into the wine, porridge and soup ". Published in 2013, "China functional food material basic composition data table" [11] first "common flower and pollen raw materials" single chapter, clearly pointed out that many flowers are used in a traditional herbal medicine and hsve modern nutrition significance. Bright colored flowers with fragrance, very pretty and charming, since ancient times familiar as ornamental products, and praised in poetry. Not only for medicine into clinical treatment, but also acid lotion, do wine bath, incense pillow for makeup, it is not only an integral part of the daily life, but 
also the traditional Chinese culture is an important [12]. Root and rhizome parts have its medical value in the treatment of disease, the modern studies spent most of active ingredients have nutritional value, medicinal value, aesthetic value, and gradually discovered, many functional products have also been developed market, for example, pollen health food, functional beverages, flower oil or water, flowers, flowers and health-care pillow containing herbal medicine etc..

With the development of productivity, the application of flowers, medicinal herbs and flowers of root and rhizome medicinal plants was neglected once. This undoubtedly promotes the research, exploration and development of flowers. Medicinal parts for root and rhizome of medicinal plants in the growth process, the whole plant flowers occupy all the nutrients, in the modern production process, the medicinal plants do not enter into or delayed reproductive growth, nutrient consumption or consumption, so we often use disbudding and remove the flower stalks and other measures to make the nutrient focus on the supply of root and Rhizome Growth, so that the root and rhizome yield and improve the quality of [13]. The flowers of medicinal plants are mostly discarded, not only polluting the environment, but also a great waste of resources.

Some modern studies of root and rhizome of medicinal plant flowers application, have health care of socialist economic development and the people's significant role in the promotion and protection, which has social, economic and ecological benefits of [14-16] obviously. The application of root and rhizome of plant flowers is the best and is the largest of respect for nature. These are not only full use of resources, but also bring enormous economic value [17-18]. It is the full exploitation and utilization of medicinal resources and the great protection of environment [19-20]. This provides a theoretical basis for the development and utilization of medicinal resources of roots and rhizomes. Roots and rhizomes of flowers have broad application prospects in our country, and the future research should pay attention to their comprehensive application, which is of great significance for their resource development and utilization, the solution of resource shortage and economic development.

\section{ACKNOWLEDGMENT}

Foundation project: national international cooperation base (2016-65), Central Plains scholar (162101510003), Henan industry university research project (142201310013172107000012)

\section{REFERENCES}

[1] Kang Chuanzhi, Zhou Tao, Jiang Wei, Huang Luqi, Guo Lanping. Study on the standard model of commodity specifications for Chinese medicinal herbs [J]. Chinese Journal of traditional Chinese medicine, 2016, (05): 769-775.

[2] Three dingliwei city medicine rhizome medicines procurement trend analysis [J]. special economic animal and plant, 2014, (11): 19-23.

[3] Cao Lihua, Miao Ming, three. Study on medicinal value of Salvia miltiorrhiza Bunge $[\mathrm{J}]$. Chinese Journal of traditional Chinese medicine, 2017, (04): 616-618.

[4] Yajiao Zhuang, Qi Chengcheng, Qian Wenxiang, wearing green,
Huang Jinni, Deng le. Seven kinds of flower pollen microscopic identification of crude drug [J]. Education Forum, 2015, (39): 250-251.

[5] Liu Aping, Cheng Huyin, Shao Haozhen, Wang Linan. Take the traditional Chinese medicine resistance of [J]. modern Chinese medicine, 2015, (04): 64-65.

[6] Liu Ying, Wu Daquan. Analysis of common medicinal plants in Laoshan [J]. Shandong forestry science and technology, 2015, (06): 60-65.

[7] Zuo Yuri Huang, Wang Liting, Chen Shouqiang. Application of flower medicine in cardiovascular diseases [J]. Chinese Journal of integrated traditional and Western medicine and cardiovascular diseases, 2016, (12): 1430-1431.

[8] what Qingyan. 10 kinds of edible Chinese herbal medicine of exogenous toxic and harmful substances residue of [D]. Hunan University of Chinese Medicine, 2016.

[9] Wang Guangming, Chen Yuqian, Liu Qian. The application of flower medicine in regulating the Qi and Yang of the spleen and stomach qi $[\mathrm{J}]$. Chinese Journal of traditional Chinese medicine, 2015, (02): 176-177+180.

[10] Chinese Pharmacopoeia. 1. [S]. 2015.

[11] Liu Bing. Study on the application of flower Materia Medica in conservation therapy [D]. Guangzhou University of Chinese Medicine, 2015.

[12] Tan Dongming, Shi Xiangli, Luo Xing Ye, Nongyan Xian, Liang Wei, Huang Jian. Guangxi Tiancha chemical composition, extraction and biological activity of $[\mathrm{J}]$. medicine, 2017, (06): 1252-1255.

[13] Xian Jing, Fu Jiang, Cheng Jintang, Jiao Mengjiao, Wang Shuhui, Zhang Jun, Liu. On the chemical constituents of medicinal rhubarb [J]. China experimental pharmacology journal, 2017, (14): 45-51.

[14] Niu Jiangtao, Cao Rui, Yang Tao, Li Yuefeng. Survey of traditional Chinese medicine resources experience some of the traditional Chinese medicine resources protection and sustainable use of the thinking of $[\mathrm{J}]$. lishizhen medicine and traditional Chinese medicine, based on the 2017 (03): 700-701.

[15] Zhang Lidong, Gong Ji Yu. Protection and sustainable utilization of traditional Chinese medicine resources [J]. World News Digest of medical information, 2016, (18): 203-204.

[16] Liu Xia, Hu Zhigang, Du Wei, Xu Lei, Duan Baozhong, Li Juping, Xie Caixiang, Zhang Xiaocun, fan Jia, Wang Jun, Chen Shilin. Shennongjia traditional Chinese medicine resource protection status and sustainable utilization strategy of [J]. world science and technology modernization of traditional Chinese medicine, 2014, (11): 2331-2336

[17] Haas bagen. Inner Mongolia Hexigten Banner Mongolian folk medicinal wild plants in the preliminary investigation of doctor [J] lishizhen medicine and Chinese medicine, 2017, (05): 1204-1206.

[18] Li Dandan, Jiang Wei, Qinling Mountains, Hu Jun, Tian Yelin. Beijing common medicinal plant resources and its utilization of [J]. China Agricultural Science Bulletin, 2016, (16): 105-109.

[19] of Guo Sheng, Su Epidendrum, Qian Dawei, Shang Er Xin, Zhou Wei, Liu Pei, Tang Yuping, Wu Qinan, Ding Anwei. The process of industrialization of Chinese medicine resources waste and resource utilization and Strategies of herbs, model [J]. 2013, (20): 2787-2797.

[20] Zhang Xiaoyan, Gong Wei, Zhang Tiejun, Chen Changqing. Research status of reutilization of medicinal plant waste [J]. Chinese herbal medicine, 2016, (07): 1225-1229. 